\title{
Crack Growth Direction in Mixed Mode Loading: A Strain Energy Density Approach
}

\author{
Hasnaa W. Taha, Tawakol A. Enab*, M. A. N. Shabara, Ahmed M. Galal \\ Production Engineering and Mechanical Design Department, Faculty of Engineering, Mansoura University \\ P.O. 35516 Mansoura, Egypt. \\ * Corresponding author's email: tenab [AT] mans.edu.eg
}

\begin{abstract}
The crack growth in metallic materials using fast and reliable simulations of 2-D and linear elastic finite element models is investigated. The effect of the stress intensity factor in mode I and II (KI, KII) on the fracture behavior of stainless steel and the associated strain energy density factor in mixed mode crack propagation were studied numerically to determine crack propagation angle $\theta$ in linear elastic fracture investigation. In order to implement the determination of the crack propagation direction using the strain energy density criterion $S$, the numerical finite element program ANSYS was used. ANSYS APDL macros were developed to generate the geometry, material properties, boundary conditions and mesh size of the model for the conducted analyses. To demonstrate the capability of crack propagation trajectories using the proposed method under mixed mode situation, an edge crack specimen was considered with initial crack having the same length but at different inclination angles under a uniaxial tension load. Results obtained from the developed models had a good agreement (average deviation of 4.63\%) with the results available in the literatures.
\end{abstract}

Keywords_- Mixed mode Fracture; Crack propagation; Strain energy density; Finite element method (FEM).

\section{INTRODUCTION}

Cracks can cause structures to fail, so it is very important to study how cracks behave, even in small or large mechanical components. If a large structure, such as a building or a ship, cracks, this may lead to a failure which could be catastrophic. So, the analysis of crack propagation and failure prediction in the structural components are very important issues to research. Nowadays, the numerical analysis of fracture problems has become an effective way of approaching this problem because of the development of the computing capacity.

Inglis, in 1913, wrote the first significant paper in which he made the analytical formulation of stresses in the vicinity of a crack tip[1]. In his famous paper [2] Griffith, Using Inglis' work as a foundation, he devised an energy-rate analysis of equilibrium of cracks in brittle materials[3] His first work that was devoted to fracture formed the foundation of modern fracture theory. The principles of linear elastic fracture mechanics (LEFM) were developed in the 1950s by George Irwin. This work was based on previous investigations of Griffith (1920) and Orowan (1944) [4]. Irwin stressed out that any fracture can be described in terms of the three fundamental modes of fracture: I. the symmetrical opening mode, II. the sliding mode and III. the tearing mode[5]. Since these first studies, the research in linear elastic fracture mechanics (LEFM) has led to the development of a vast number of theories and applications.

The successful experiments in 1971 by Begley and Landes [6], led to the publication of a standard procedure for testing of metals in 1981. Sih [7] theoretically analyzed the strain-energy density field near the crack tip under multiaxial loading, which was a departure from classical fracture mechanics. In this paper, the strain-energy-density factor criterion is applied to the problem of biaxial tension of plates containing single U-notched edge cracks oriented at different crack inclination angles.

\section{STRAIN-ENERGY-DENSITY CRITERION (S-CRITERION)}

Most of the previous theoretical investigations on crack initiation and propagation have been concerned with mode I crack-tip loading. The strain energy density factor, $S$, of Sih is a quantity evaluated at any point on an arc located at a radius $r$ away from the crack tip and is usually applied to crack tip singularities. Because it is a function of $\theta$, a minimum 
value of $S$ can be found at a given angle $\theta$. Thus, the strain energy density factor may be used as a criterion for predicting the crack propagation direction, as well as a failure criterion [8]. The variations of the minimum values of $S$, which is a function of the stress intensity factors for linear elasticity, along the crack border will be studied in order to apply the strain energy density theory to crack problems in two dimensions. The fracture criterion developed by Sih [7], the critical strain energy density factor, postulates that a crack that initiate when, and in a direction where, the strain energy density factor, $S$, assumes a critical value, $S_{c}$, around the crack tip. The strain energy density factor is found from the following expression [7].

$$
S=\mathrm{a}_{11} \cdot \mathrm{k}_{I}^{2}+2 \mathrm{a}_{12} \cdot \mathrm{k}_{I} \cdot \mathrm{k}_{I I}+\mathrm{a}_{22} \cdot \mathrm{k}_{I I}^{2}
$$

where KI and KII are the stress intensity factors under loading modes I and II, respectively, and the coefficients $a_{i j}$ for plane strain condition are given by:

$$
\begin{aligned}
& a_{11}=\frac{1}{16 \mu}[(3-4 v-\cos \theta)(1+\cos \theta)] \\
& a_{12}=\frac{1}{16 \mu}(2 \sin \theta)[\cos \theta-(1-2 v)] \\
& a_{22}=\frac{1}{16 \mu}[4(1-v)(1-\cos \theta)+(1+\cos \theta)(3 \cos \theta-1)]
\end{aligned}
$$

with $v$ being the Poisson's ratio of the material and $\mu$ the shear modulus of elasticity [9].

The angle $\theta$, which is measured from the local crack direction, corresponding to the minimum of $S$ can be determined from the first and second derivatives of Eq. (1) with respect to $\theta$. According to the strain energy density function, crack initiate and propagate when:

$$
\begin{aligned}
& \frac{\partial S}{\partial \theta}=0 \\
& \frac{\partial^{2} S}{\partial \theta^{2}}>0 \quad \text { at } \theta=\theta_{o}
\end{aligned}
$$

where $\theta$ is the polar angle. Crack initiation occurs when [10]:

$$
S\left(\theta_{o}\right)=\mathrm{S}_{c}
$$

and substituting S from Eq. (1) we obtain

$$
\begin{aligned}
& {[2 \cos \theta-(\kappa-1)] \sin \theta \mathrm{K}_{I}^{2}+2[2 \cos 2 \theta-(\kappa-1) \cos \theta] \mathrm{K}_{I} K_{I I}} \\
& +[(\kappa-1-6 \cos \theta) \sin \theta] \mathrm{K}_{I I}^{2}=0 \\
& {[2 \cos 2 \theta-(\kappa-1) \cos \theta] \mathrm{K}_{I}^{2}+2[(\kappa-1) \sin \theta-4 \sin 2 \theta] \mathrm{K}_{I} K_{I I}} \\
& +[(\kappa-1) \cos \theta-6 \cos 2 \theta] \mathrm{K}_{I I}^{2}>0
\end{aligned}
$$

where $\kappa=3-4 v$ for plane strain condition.

Relations (5.a) and (5.b) represent the general formulae of the strain energy density criterion for the case of a crack in a two-dimensional stress field under mixed-mode loading conditions [11].

Because of the stress singularity at the crack tip, obtaining an accurate stress intensity factor from the stress field involves very tedious and time-consuming computation. Advances in numerical techniques, such as finite element method, allows relatively rapid determination of the displacement field in the region close to the crack tip with good accuracy[12].

\section{NUMERICAL SIMULATION MODEL}

In this paper, the case of single edge crack in a plate of finite width subjected to a uniform uniaxial stress will be studied. The crack will be oriented at various inclination angles $(\alpha)$, Figure 1, which generally produces mixed-mode 
conditions at the crack tips depending on the strain energy density criterion by means of finite element method (FEM) that provides a reasonable way to predict the failure behavior of materials.

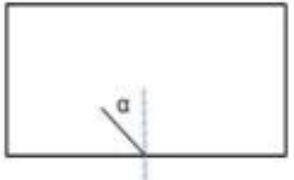

(a)

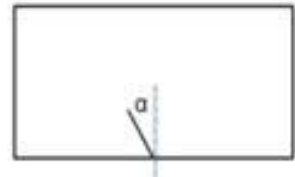

(b)

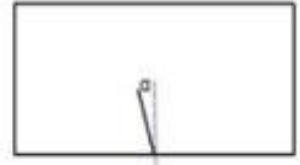

(c)

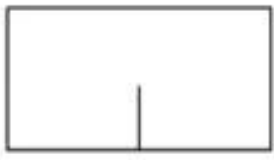

(d)

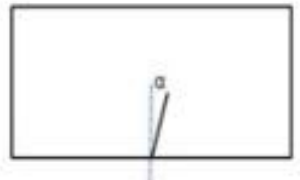

(e)

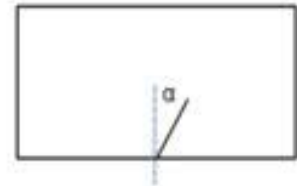

(f)

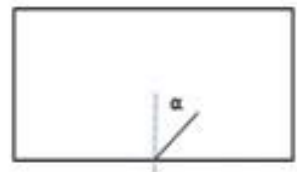

(g)

Figure 1: The crack angle $\alpha$ with respect to the vertical plane was set to be: (a) $45^{\circ}$, (b) $30^{\circ}$, (c) $15^{\circ}$, (d) $0^{\circ}$, (e) $-15^{\circ}$, (f) $30^{\circ},(\mathrm{g})-45^{\circ}$.

\section{FINITE ELEMENT PROCEDURE}

A macro is written using ANSYS APDL[13] to generate the geometry, material properties, boundary conditions and mesh size of the model for the conducted analyses. This ANSYS input code is written in terms of crack notch angle $(\alpha)$, crack notch radius $(\rho)$ and pre-crack length $(a)$ in order to change the variables easily.

Consider the linear elastic plate represented in figure (2) its dimensions are the width $W=50 \mathrm{~mm}$, the length $L=100$ $\mathrm{mm}$, the thickness $t=10 \mathrm{~mm}$. The crack is placed perpendicular to the loading direction in the center of the lower half of the plate and has a length of $a=2 \mathrm{~mm}$ and radius of curvature $\rho=0.035 \mathrm{~mm}$. The edge-cracked tension plate is assumed to be in the plane strain condition in this analysis.

Stainless steel 304 [14] was modeled as a linear isotropic material with elastic modulus $E=210 \mathrm{GPa}$ and Poisson's ratio $v=0.3$. In order to obtain a better approximation of the field near the crack tip, an isoperimetric eight or six nodes quadratic or triangle displacement behavior element is used which is better suited to modeling irregular meshes [15].

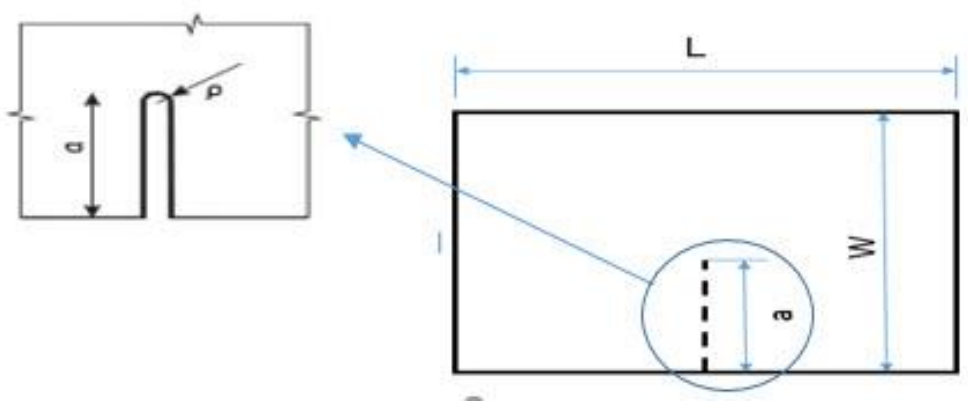

Figure 2: Configuration of single edge crack specimen.

In general, to obtain more accurate finite element approximate solution, a smaller mesh size is required. However, the reduction in the mesh size leads to greater computational time. In the fracture mechanics problems, the initial mesh generation is a key step. Two dimensional geometries that containing cracks are difficult and complex to mesh because the vicinity near the crack tip needs to be very fine meshed while a coarse mesh up to two orders of magnitude larger will suffice the area that is far from the crack tip.

In the current study, a linear elastic fracture model is considered but the used methodology can be extended for more complex models. The growth direction of the crack is computed using a finite element model for each new kink crack. The mesh configuration from ANSYS for the numerical determination of the crack inclination angle is shown in Figure 3.

In the case of an inclined single edge crack, the model was not symmetric. Thus, full model of the edge cracked plate was analyzed using ANSYS environment. In this study, it was assumed that the plate was fixed at the left vertical boundary and the uniform tensile stress $\sigma$, which is applied at the right vertical boundary of the plate, is large enough so that the studied fracture criterion holds as shown in Figure 4. In this way, it is known that the initial crack will propagate. 
The remaining question is, in which direction the crack will propagate? The answer of this question is the goal of this study.

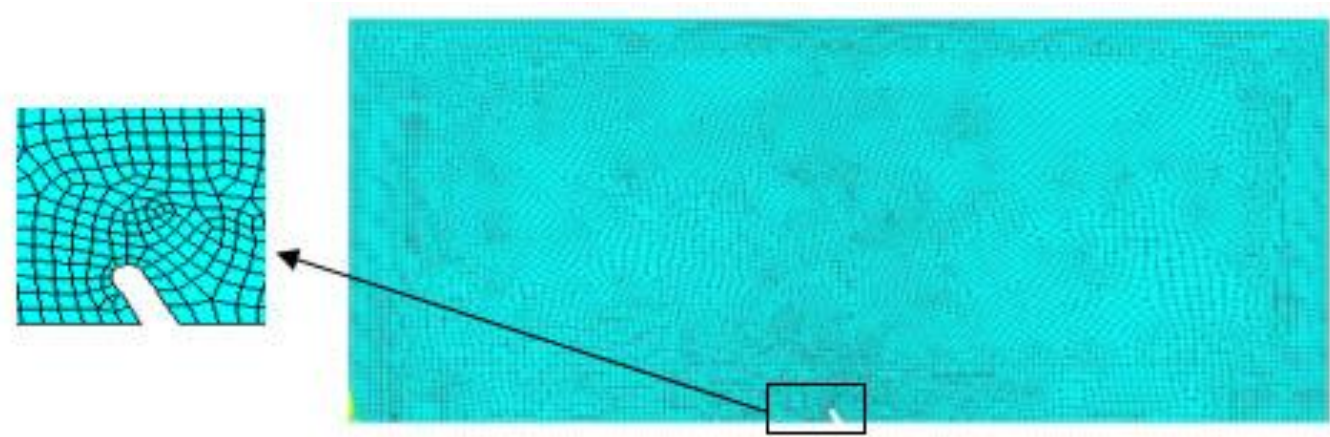

Figure 3: Mesh configuration of an inclined single edge U-notched kink crack specimen.

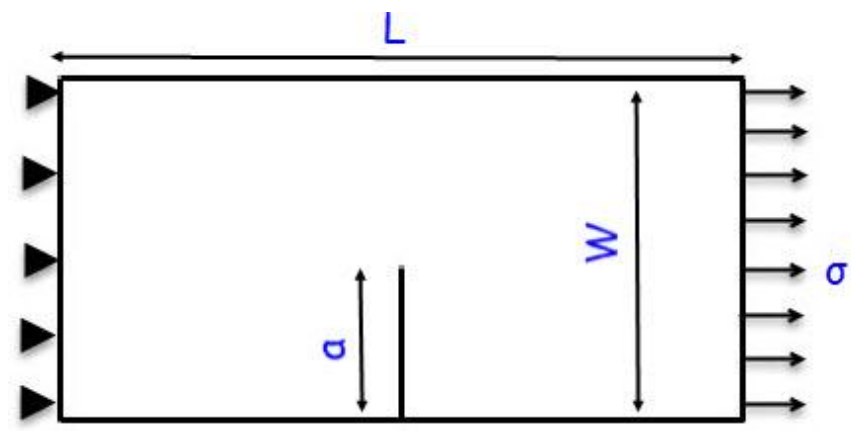

Figure 4: Schematic of the boundary conditions of single-edge-notch plate in tension.

\section{RESULTS AND DISCUSSION OF NUMERICAL SIMULATION}

Since the analysis of the current research is entirely based on finite element modeling and its results, it is considered that the verification of the developed finite element models is essential. Thus, the developed finite element models were initially verified and then used to predict the crack propagation trajectory of single-edge-notched plate under tension load at different inclination angles in metallic materials. The developed models with a little modification can handle a wide variety of different materials, dimensions and boundary conditions. The only limitation is imposed by the available computational resources.

The verification analysis was performed by using the developed FE models for certain cases in the literature and reproducing their results at a crack inclined to the horizontal with the angle $\alpha=20^{\circ}[16]$ as shown in Figure 5. Therefore, the aluminum alloy with modulus of elasticity $E=72.4 \mathrm{GPa}$ and Poisson's ratio $v=0.3$ is considered for the modeling of the isotropic material plate.
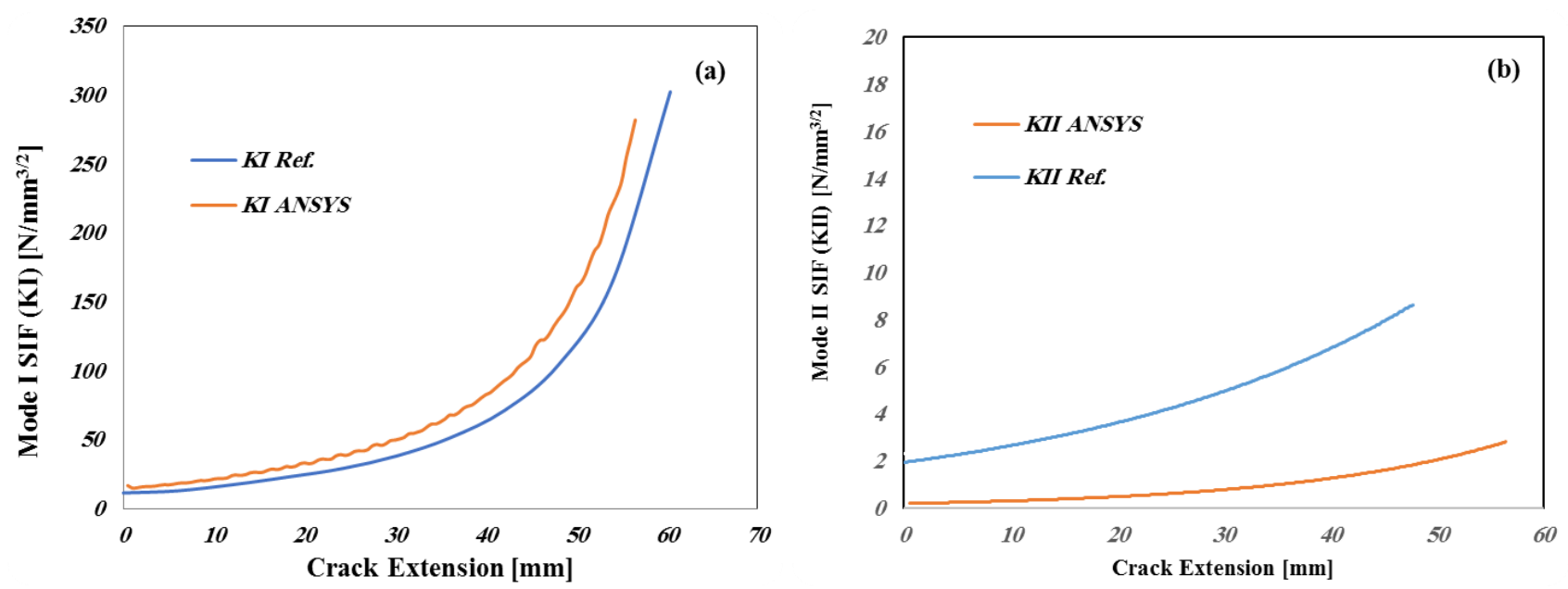

Figure 5: Comparison between the FE results and reference [16] of the stress intensity factor values (a) Mode I, (b) Mode II. 
To determine at which angle the predicted crack will propagate from the crack tip (crack propagation angle $\theta$ ) numerically on the basis of strain energy density factor $(S)$, the values of the stress intensity factors KI and KII at different crack inclination angle, $\alpha$, are determined along the crack path. The accurate evaluation of the stress intensity factors of a crack under mixed mode loading is relevant in order to establish the crack propagation angle. The obtained $\mathrm{KI}$ and KII values from the finite element models were plotted for U-notched kink crack specimens.

Numerical evaluation of stress intensity factors (SIFs) for interface notches is important, since cracks or fractures are often initiated at these locations[15]. As shown in Figure (6-a) the numerical values of the stress intensity factor in mode I, KI, are obtained along different kink angles demonstrated that KI increases along the crack path until fracture. it is close to its maximum value along the direction of theoretical initiation angle. while it has inconsiderable changes at different crack inclination angles $\alpha$. SIF, KI, values are almost the same for $\alpha-45^{\circ}$ and $45^{\circ}, \alpha-30^{\circ}$ and $30^{\circ}, \alpha-15^{\circ}$ and $15^{\circ}$ and they are all around the values of $\alpha$ zero. That means the crack inclination angle, $\alpha$, has no significant influence on the stress intensity factor values in the biaxially loaded single edge U-Notched plate.

Figure (6-b) represents the variation of the numerical values of the stress intensity factor KII, obtained along different inclination angles demonstrated that KII has very small values close to zero. Theoretically, in the case of vertical kink crack ( $\alpha$ equal zero) KII should be zero this can be explained by the fact that the whole plate was modelled and some nodes are not laid on the crack plane.

Figure 7 shows the numerical values of the strain energy density factor obtained along different inclination angles, the figure demonstrates that $S$ increases along the crack path until it reached fracture. The factor has its minimum value (critical value $S_{c}$ ) at each kink along crack path. Changing the crack inclination angle $\alpha$ seems to have slight effect, almost no significant effect, on the crack direction.

FE simulation results illustrate the relation between crack propagation angle $\theta$ and the strain energy density factor $S$ along the crack path using single-edge-U-notched beam at different crack inclination angles $\alpha$. From the plotted figures, the crack can be reasonably assumed to propagate in the direction predicted after the crack initiated in a straight line. This implies that after the crack initiates, crack propagation is mainly dominated by mode I for the present testing configuration. The only major difference on these values is the crack initiation angle, $\theta_{o}$, which is mainly affected by the crack inclination angle.
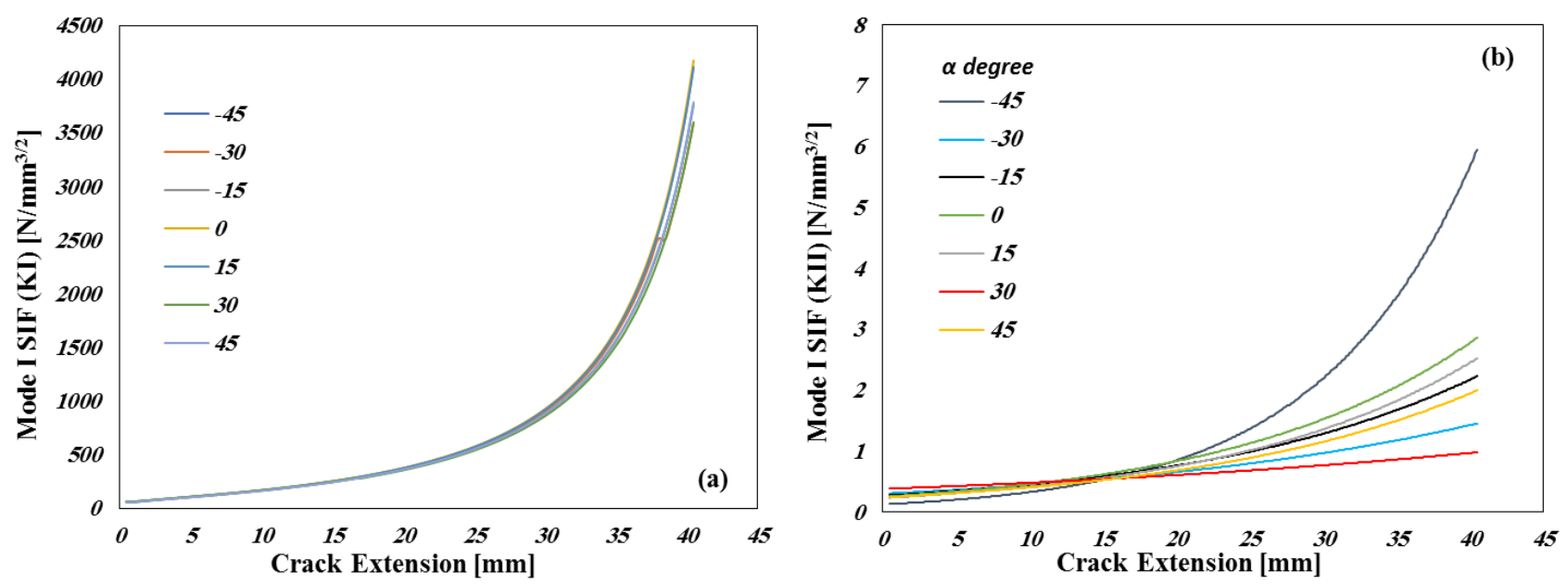

Figure 6: Schematic of the stress intensity factor values in (a) mode I and (b) mode II of single-edge-U-notched plate in tension. 


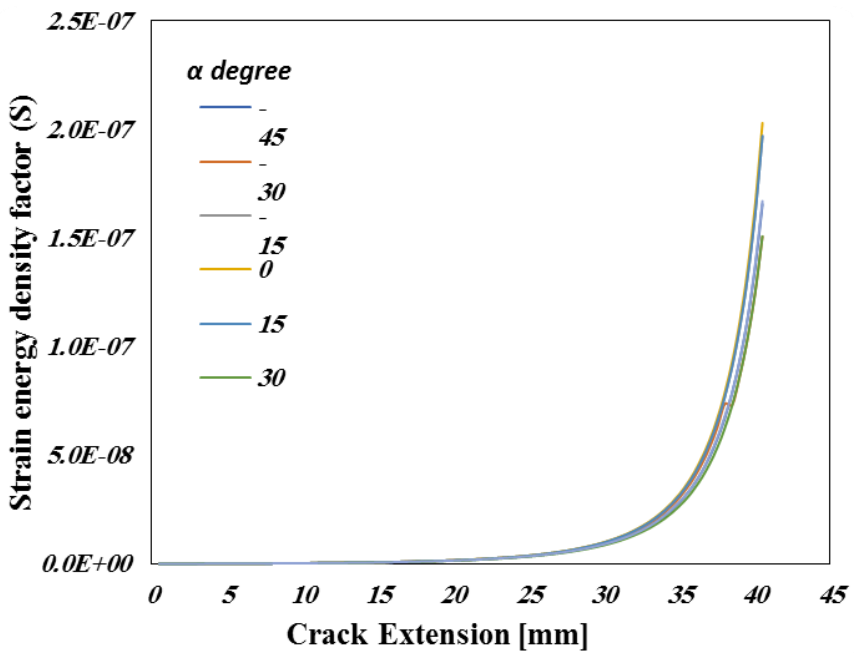

Figure 7: Schematic of the strain energy density factor values in biaxial tension loading of single-edge-U-notch plate.

\section{CONCLUSION}

A two-dimensional finite element model was developed ANSYS APDL macro for crack growth analysis, using to examine the crack propagation of stainless steel plates with a U-notched crack at various pre-crack inclination angles under uniform tension load are analyzed.

The numerical analysis of the present work showed the stress intensity factors for mode I and II, the strain energy density factor and the crack propagation angle along path trajectories of the propagated crack.

the crack inclination angle, $\alpha$, has no significant influence on the stress intensity factor values in the biaxially loaded single edge U-Notched plate therefore it seems to have slight effect, almost no significant effect, on the crack direction.

The numerical results of the SIF indicate that KI is the parameter that controls the process of the crack propagation even in the case of biaxial loaded specimens. After the crack initiates, crack propagation is mainly dominated by mode I for the present testing configuration.

The only major difference due to the crack inclination angle is in the crack initiation angle, $\theta_{\mathrm{o}}$, which is mainly affected by the crack inclination angle and the properties of the used material.

\section{REFERENCES}

[1] C. E. Inglis, "Stresses in a plate due to the presnce of cracks and sharp corners," Trans. Inst. Naval Arch., vol. 55. pp. 219-239, 1913.

[2] A. A. Griffith, "The Phenomena of Rupture and Flow in Solids," 1920.

[3] P. C. Paris and G. C. Sih, "Stress analysis of cracks," ASTM International, Bethlehem, Pennsylvania, 1964.

[4] J. J. Mecholsky, "Fracture mechanics principles," Dent. Mater., vol. 11, no. 2, pp. 111-112, 1995.

[5] D. K. McCarthy, "Numerical Prediction of Fracture in Composite Materials Using A Modified Strain Energy Density Criterion," 2012.

[6] J. Begley and J. Landes, “The J integral as a fracture criterion,” Astm Stp 514. pp. 1-23, 1972.

[7] G. C. Sih, Mechanics of fracture initiation and propagation. Kluwer Academic Publishers, 1991.

[8] Z. Yosibash, A. Bussiba, and I. Gilad, "Failure criteria for brittle elastic materials," Int. J. Fract., no. 1957, pp. 307333, 2004.

[9] G. C. Sih and B. Macdonald, "Fracture Mechanics Applied to Engineering Problems-Strain Energy Density Fracture Criterion," Eng. Fract. Mech., vol. vol.6, pp. 361-386, 1974.

[10]E. E. Gdoutos, Fracture Mechanics An Introduction, Second Edi. Netherlands: Springer, 2005.

[11]E. E. Gdoutos, Problems of mixed mode crack propagation. Netherlands.: Martinus Nijhoff Publishers, 1984.f mixed mode crack propagation. Netherlands.: Martinus Nijhoff Publishers, 1984.

[12]P. C. Gope, S. P. Sharma, and A. K. Srivastava, "Prediction of crack initiation direction for inclined crack under biaxial loading by finite element method," J. Solid Mech., vol. 2, no. 3, pp. 257-266, 2010.

[13]“ANSYS Engineering analysis system. Users manual.” ANSYS Inc., Canonsburg (PA), 2015.

[14] Steel Products Manual: Stainless Steels. U.S.A: Iron \& Steel Society, 1999. 
[15] S. H. Ju, "Finite element calculation of stress intensity factors for interface notches," Comput. Methods Appl. Mech. Eng., vol. 199, no. 33-36, pp. 2273-2280, 2010.

[16] A. Boulenouar, N. Benseddiq, M. Mazari, and N. Benamara, "FE Model for Linear-Elastic Mixed Mode Loading: Estimation of Sifs and Crack Propagation," J. Theor. Appl. Mech., vol. 52, no. 2, pp. 373-383, 2014. 